\title{
Coordination between Electron Transfer and Molecule Diffusion through Bioinspired Amorphous Titania Nanoshell for Photocatalytic Nicotinamide Cofactor Regeneration
}

Dong Yang, Yishan Zhang, Shaohua Zhang, Yuqing Cheng, Yizhou Wu, Ziyi Cai, Xiaodong Wang, Jiafu Shi, and Zhongyi Jiang

ACS Catal., Just Accepted Manuscript • DOI: 10.1021/acscatal.9b03462 • Publication Date (Web): 04 Nov 2019

Downloaded from pubs.acs.org on November 5, 2019

\section{Just Accepted}

"Just Accepted" manuscripts have been peer-reviewed and accepted for publication. They are posted online prior to technical editing, formatting for publication and author proofing. The American Chemical Society provides "Just Accepted" as a service to the research community to expedite the dissemination of scientific material as soon as possible after acceptance. "Just Accepted" manuscripts appear in full in PDF format accompanied by an HTML abstract. "Just Accepted" manuscripts have been fully peer reviewed, but should not be considered the official version of record. They are citable by the Digital Object Identifier (DOI®). "Just Accepted" is an optional service offered to authors. Therefore, the "Just Accepted" Web site may not include all articles that will be published in the journal. After a manuscript is technically edited and formatted, it will be removed from the "Just Accepted" Web site and published as an ASAP article. Note that technical editing may introduce minor changes to the manuscript text and/or graphics which could affect content, and all legal disclaimers and ethical guidelines that apply to the journal pertain. ACS cannot be held responsible for errors or consequences arising from the use of information contained in these "Just Accepted" manuscripts. 


\title{
Coordination between Electron Transfer and Molecule Diffusion through Bioinspired Amorphous Titania Nanoshell for Photocatalytic Nicotinamide Cofactor Regeneration
}

\author{
Dong Yang,b, Yishan Zhang ${ }^{\mathrm{b}, \mathrm{c}}$, Shaohua Zhang ${ }^{\mathrm{b}, \mathrm{c}}$, Yuqing Cheng ${ }^{\mathrm{b}, \mathrm{c}}$, Yizhou $\mathrm{Wu}^{\mathrm{b}, \mathrm{c}}$, \\ Ziyi Cai ${ }^{\mathrm{b}, \mathrm{c}}$, Xiaodong Wange, ${ }^{\mathrm{e},}$, Jiafu Shi ${ }^{\mathrm{a}, \mathrm{c},{ }^{*}}$ and Zhongyi Jiang ${ }^{\mathrm{b}, \mathrm{c}, \mathrm{d},{ }^{*}}$ \\ a School of Environmental Science and Engineering, Tianjin University, 92 Weijin \\ Road, Nankai District, Tianjin 300072, P. R. China \\ ${ }^{\mathrm{b}}$ Key Laboratory for Green Chemical Technology of Ministry of Education, School of \\ Chemical Engineering and Technology, Tianjin University, 92 Weijin Road, Nankai \\ District, Tianjin 300072, P. R. China \\ ${ }^{c}$ Collaborative Innovation Center of Chemical Science and Engineering (Tianjin), 92 \\ Weijin Road, Nankai District, Tianjin 300072, P. R. China \\ d State Key Laboratory of Biochemical Engineering, Institute of Process Engineering, \\ Chinese Academy of Sciences, 1 North 2nd Street, Zhongguancun, Haidian District, \\ Beijing, 100190, P. R. China \\ e Chemical and Materials Engineering, School of Engineering, University of \\ Aberdeen, Aberdeen AB24 3UE, Scotland, UK \\ ${ }^{*}$ Corresponding authors:
}

Jiafu Shi, shijiafu@tju.edu.cn; Zhongyi Jiang, zhyjiang@tju.edu.cn 


\begin{abstract}
In-depth understanding and rational manipulation of the electron transfer process and molecule diffusion process are critical to promote the overall photocatalytic efficiency. In our study, core@shell photocatalysts that embody graphitic carbon nitride $(\mathrm{GCN})$ core and amorphous titania $\left(\mathrm{a}-\mathrm{TiO}_{2}\right)$ nanoshell are prepared to elucidate and coordinate the electron transfer and molecule diffusion for the regeneration of nicotinamide adenine dinucleotide $(\mathrm{NADH})$ with $\left[\mathrm{Cp} * \mathrm{Rh}(\mathrm{bpy}) \mathrm{H}_{2} \mathrm{O}\right]^{2+}$ as the redox mediator. The GCN core absorbs visible light to generate electron-hole pairs, whereas the a- $\mathrm{TiO}_{2}$ nanoshell facilitates the transfer of photo-generated electrons from $\mathrm{GCN}$ to the $\mathrm{a}-\mathrm{TiO}_{2}$ surface for NADH regeneration, which also enables the diffusion of electron donor molecules (TEOA) from the $\mathrm{a}^{-\mathrm{TiO}_{2}}$ surface to $\mathrm{GCN}$ for consuming the holes left on GCN. The transfer of photo-generated electrons and the diffusion of electron donor molecules are coordinated by finely tuning the thickness of a- $\mathrm{TiO}_{2}$ nanoshell. Under the optimized nanoshell thickness of $2.1 \mathrm{~nm}$, the GCN@a-TiO photocatalyst exhibits the highest NADH regeneration yield of $82.1 \%$ after 10 -min reaction under LED light (405 nm), over 200\% higher than GCN photocatalyst. Combined with the highly controllable and mild features of the bioinspired mineralization method, our study may offer a facile and generic strategy to design high-performance photocatalysts through rational coordination of different substances/species transport processes.
\end{abstract}

Keyword: Coordination; Electron Transfer; Molecule Diffusion; Amorphous Titania Nanoshell; Bioinspired mineralization; Photocatalytic NADH Regeneration 


\section{Introduction}

Coordinating the transfer/diffusion processes of multiple substances, e.g., electrons, ions, molecules, is critical for the efficient energy conversion in nature. Understanding and exploring the coordinated mechanism would help to elevate the energy conversion efficiency and sustain the development of modern society. Photocatalysis is a green energy conversion process, which can directly convert solar energy into fuels and valuable chemicals. ${ }^{1-4}$ A photocatalytic reaction commonly includes three primary processes: photo-generated charge carrier transfer, reactant molecule diffusion, as well as reaction between carriers and molecules. ${ }^{5-7}$ Individual intensification of each process and coordinated optimization of all three processes are both essential for enhancing the overall process efficiency.

The intensification of photo-generated charge transfer is frequently used to promote photocatalytic performance, which can be realized by engineering the band structures, physical structures or hetero-structures of photocatalyst. ${ }^{8-12}$ Amongst, core@shell hetero-structures have sparked numerous interests because of the flexible and independent regulation of crystal faces/forms/materials of the core and the shell. ${ }^{13-16}$ In this regard, tremendous efforts have been devoted to prepare core@shell hetero-structured photocatalysts, including transition metal oxyhydroxides@ $\mathrm{SrTiO}_{3}$, CoO ${ }_{\mathrm{x}} @ \mathrm{Ta}_{3} \mathrm{~N}_{5}, \mathrm{MOF} @ \mathrm{COF}, \mathrm{CdS} @ \mathrm{TiO}_{2}, \mathrm{Au} @ \mathrm{TiO}_{2}$, etc. ${ }^{14,17-20}$ As for a core@shell hetero-structured photocatalyst, the core material generally acts as a light harvester to induce electron-hole separation, while the shell material facilitates the electron transfer from the core material surface. ${ }^{21-23}$ The incorporation of shell material can 
effectively inhibit electron-hole recombination and elevate photocatalytic conversion efficiency up to $99 \%$ for hydrogenation reaction. ${ }^{18}$ It is also reported that shell thickness is closely related electron transfer behavior, where a thicker shell with intact structure and transparent feature can lead to higher interfacial electron transfer rate. ${ }^{20}$ Albeit significant progresses have been achieved in designing core@shell photocatalysts to facilitate the charge transfer, the coordination between charge transfer and molecule diffusion was rarely reported, which also seriously restricted the photocatalytic performance since the shell would frequently inhibit the diffusion of reactant molecules. In theory, a thicker shell is expected to cause decreased diffusion rate of electron donor molecules, which further results in lower photocatalytic efficiency. Then, the shell thickness of a core@shell hetero-structured photocatalyst shows a "trade-off" effect in balancing the two processes of photo-generated electron transfer and electron donor molecule diffusion, which may determine the final photocatalytic efficiency. Currently, rare investigations concern such a topic probably due to the difficultly in acquiring intact, porous and conductive shell on a core material. Therefore, developing a method to prepare core@shell photocatalyst with intact, porous and conductive shell would construct a platform to coordinate photo-generated electron transfer and electron donor molecule diffusion, and finally promote the overall photocatalytic efficiency.

Herein, for the first time, a bioinspired mineralization method is developed to form an intact, porous and conductive of amorphous titania $\left(\mathrm{a}-\mathrm{TiO}_{2}\right)$ nanoshell on graphitic carbon nitride $(\mathrm{GCN})$ core for the preparation of $\mathrm{GCN} @ \mathrm{a}-\mathrm{TiO}_{2}$ core@shell 
photocatalyst. ${ }^{24} \mathrm{GCN}$, a metal-free semiconductor that has been successfully used for NADH regeneration, was chosen as the model core to harvest visible light and generate electron-hole pairs. ${ }^{25}$ The a- $\mathrm{TiO}_{2}$ nanoshell facilitates the transfer of photo-generated electrons from $\mathrm{GCN}$ to the a- $\mathrm{TiO}_{2}$ surface for the regeneration of nicotinamide adenine dinucleotide (NADH) with $\left[\mathrm{Cp} * \mathrm{Rh}(\mathrm{bpy}) \mathrm{H}_{2} \mathrm{O}\right]^{2+}$, and at the same time enables the diffusion of electron donor molecules (TEOA) form the $\mathrm{a}-\mathrm{TiO}_{2}$ surface to GCN for consuming the holes left on GCN. ${ }^{26}$ The thickness of the $\mathrm{a}-\mathrm{TiO}_{2}$ nanoshell could be controlled through changing the mineralization-inducer concentration and the mineralization cycles, which could optimally coordinate the photo-generated electron transfer process and electron donor molecule diffusion to pursue high photocatalytic efficiency. Our GCN@a-TiO 2 core@shell photocatalyst with controlled nanoshell thickness could establish a platform for understanding the coordination mechanism between molecule diffusion and electron transfer. When coupling the photocatalytic NADH regeneration with enzyme catalysis, the nanoshell could effectively isolate photo-generated holes and enzyme, which remarkably improved the compatibility between photocatalyst and enzyme. Moreover, the bioinspired mineralization method is performed under ambient conditions, e.g., room temperature, aqueous solution and neutral $\mathrm{pH}$ value, ${ }^{27}$ which is applicable for producing a broad range of core@shell photocatalysts based on different core materials.

\section{Experimental section}

\section{Materials}


Melamine, triethanolamine (TEOA) and pentamethylcyclopentadienyl rhodium (III) chloridedimer $\left(\left(\mathrm{Cp}^{*} \mathrm{RhCl}_{2}\right)_{2}\right)$ were purchased from Shanghai Aladdin Co., Ltd. $\beta$-nicotinamide adenine dinucleotide phosphate sodium salt hydrate $\left(\mathrm{NAD}^{+}\right)$, protamine sulfate salt from salmon, titanium (IV) bis (ammonium lactato) dihydroxide solution (Ti-BALDH, $50 \mathrm{wt} \%$ aqueous solution) and yeast alcohol dehydrogenase (YADH) from Saccharomyces cerevisiae were purchased from Sigma-Aldrich Co., Ltd. Methanol was obtained from Tianjin Kemiou Chemical Reagent Co., Ltd. Potassium sodium tartrate was got from Shanghai Yuanye Biotechnology Co., Ltd. All other reagents were used without further purification.

\section{Preparation of Bulk GCN}

Bulk GCN was prepared through a one-step thermal condensation of melamine. The heating temperature was increased to $550^{\circ} \mathrm{C}$ from room temperature with a ramp rate of $5{ }^{\circ} \mathrm{C} \mathrm{min}^{-1}$, and then kept at $550{ }^{\circ} \mathrm{C}$ for 4 hours. ${ }^{28}$ After cooling down to the room temperature, bulk GCN (denoted as GCN) was obtained. Note that the yield of GCN was about $58.1 \%$.

\section{Preparation of GCN@a-TiO 2 Core@Shell Photocatalyst}

The GCN@a-TiO 2 core@shell photocatalyst was prepared through a bioinspired mineralization method under mild conditions. ${ }^{24}$ First, the as-prepared GCN was added into $2 \mathrm{mg} \mathrm{mL}-1$ protamine solution and shaken for $10 \mathrm{~min}$. After centrifugation and water washing, the protamine-absorbed GCN was dispersed into Ti-BALDH solution (1.25 wt $\%)$ and shaken for $10 \mathrm{~min}$. After centrifugation and water washing, GCN coated with one layer of a- $\mathrm{TiO}_{2}$ was obtained, which was denoted as $\mathrm{GCN} @ \mathrm{a}-\mathrm{TiO}_{2}-1$. 
Notably, the lower mineralization-inducer concentration $\left(0.5 \mathrm{mg} \mathrm{mL}^{-1}\right)$ was also used to prepare the GCN@a-TiO 2 core@shell photocatalyst, as well, the process of bioinspired mineralization with standard mineralization-inducer concentration $(2 \mathrm{mg}$ $\mathrm{mL}^{-1}$ ) was repeated one to two more times to control the amount of a-TiO ${ }_{2}$ on GCN. The obtained samples were denoted as GCN@a-TiO ${ }_{2}-1 *, G C N @ a-\mathrm{TiO}_{2}-2$ and GCN@a-TiO $2-3$, respectively.

$\mathrm{CdS} @ \mathrm{a}-\mathrm{TiO}{ }_{2}, \mathrm{ZnO} @ \mathrm{a}-\mathrm{TiO}_{2}$ and $\mathrm{SrTiO}_{3} @ \mathrm{a}-\mathrm{TiO}_{2}$ were also prepared to validate the universality of our bioinspired mineralization method. In brief, CdS was firstly prepared by directly mixing the same volume of $10 \mathrm{mM} \mathrm{CdSO}_{4}$ and $10 \mathrm{mM} \mathrm{Na}_{2} \mathrm{~S}$. After centrifugation, water washing and lyophilization, $\mathrm{CdS}$ was obtained. $\mathrm{CdS} @ \mathrm{a}-\mathrm{TiO}_{2}, \mathrm{ZnO} @ \mathrm{a}-\mathrm{TiO}_{2}$ and $\mathrm{SrTiO}_{3} @ \mathrm{a}-\mathrm{TiO}_{2}$ were prepared with the same procedure to GCN@a-TiO 2 , just substituting GCN with $\mathrm{CdS}, \mathrm{ZnO}$ and $\mathrm{SrTiO}_{3}$.

\section{Photocatalytic NADH Regeneration}

The photocatalytic NADH regeneration was performed in a quartz reactor (light path: $1 \mathrm{~cm}$ ) with $2 \mathrm{ml}$ phosphate buffer saline (PBS) buffer (100 mM, pH 8.0) containing TEOA (15 w/v\%), $\left[\mathrm{Cp}^{*} \mathrm{Rh}(\mathrm{bpy}) \mathrm{H}_{2} \mathrm{O}\right]^{2+}$ (denoted as $\left.\mathrm{M}, 0.25 \mathrm{mM}\right), \mathrm{NAD}^{+}$ $(1 \mathrm{mM})$ and photocatalyst $\left(1 \mathrm{mg} \mathrm{mL}^{-1}\right)$ at room temperature under $405 \mathrm{~nm}$ LED lamp illumination $(300 \mathrm{~mW} \mathrm{~cm}-2)$. The reaction system was firstly incubated in darkness for 10 min. During illumination, the regenerated NADH was detected by a UV-vis spectrophotometer (U-3010, Hitachi). The concentration of NADH was calculated according the absorbance at $340 \mathrm{~nm}$ with an extinction coefficient of $6220 \mathrm{M}^{-1} \mathrm{~cm}^{-1} \cdot 25$ Moreover, the NADH regeneration experiments were also performed under different 
concentration of TEOA (3-15 $\mathrm{w} / \mathrm{v} \%)$, different light intensity $\left(50-300 \mathrm{~mW} \mathrm{~cm}{ }^{-2}\right)$ and different $\mathrm{pH}$ values (7.0-9.0).

The initial reaction rate $\left(r, \mathrm{mmol} \mathrm{g}{ }^{-1} \mathrm{~min}^{-1}\right)$ of NADH regeneration was calculated using equation (1):

$$
r=\frac{n_{t}}{m \times t}
$$

where $n_{t}$ was the amount of regenerated NADH (mmol), $m$ was the mass of photocatalyst (g), and $t$ was the reaction time (min).

The apparent quantum yield $(\%)$ of $\mathrm{NADH}$ regeneration was calculated using equation (2):

$$
\text { Apparent quantum yield }(\%)=\frac{\mu m o l \text { of electron consumed by NADH regeneration }}{\mu \text { mol of incident photons }} \times 100
$$

Notably, both the initial reaction rate and apparent quantum yield were calculated according to the initial 2-4 min reaction. Since the regeneration of one mole of NADH would consume two moles of electrons, the amount of the electrons consumed by $\mathrm{NADH}$ regeneration was twice of the amount of regenerated NADH. The amount of the incident photons were calculated according to the light intensity on the front surface of quartz reactor $\left(300 \mathrm{~mW} \mathrm{~cm}^{-2}\right)$ and wavelength of LED lamp (405 nm).

\section{Photobiocatalytic Methanol Production}

The reaction solution composed of PBS buffer (4 mL, $100 \mathrm{mM}, \mathrm{pH} 8.0), \mathrm{NAD}^{+}(10$ $\mathrm{mM}), \mathrm{M}(0.25 \mathrm{mM})$, TEOA $(15 \mathrm{w} / \mathrm{v} \%)$, photocatalyst $\left(1 \mathrm{mg} \mathrm{mL}^{-1}\right)$, YADH $(0.2 \mathrm{mg}$ $\left.\mathrm{mL}^{-1}\right)$ and formaldehyde $(40 \mathrm{mM})$ was incubated in darkness for $10 \mathrm{~min}$. Then, the reaction solution was illuminated by $405 \mathrm{~nm}$ LED lamp at a light intensity of $300 \mathrm{~mW}$ 
$\mathrm{cm}^{-2}$. The concentration of methanol was determined by Agilent $7820 \mathrm{~B}$ gas chromatography.

\section{Characterizations}

Transmission electron microscopy (TEM) images were collected on a field-emission transmission electron microscopy (Tecnai G2 F20). Elemental analysis was conducted by electron energy loss spectroscopy (EELS) elemental mappings attached to transmission electron microscopy (TEM) and energy dispersive spectroscopy (EDS) attached to scanning electron microscopy (SEM). Sample preparation for EELS mappings was similar to the TEM. The samples were dropped on the microgrid and examined after drying. X-ray photoelectron spectroscopy (XPS) was performed in a Perkin-Elmer PHI 1600 ESCA system with a monochromatic Mg $\mathrm{K} \alpha$ source. X-ray diffractometer (XRD) was measured on a Rigaku D/max $2500 \mathrm{~V} / \mathrm{PC}$ instrument with the graphite filtered $\mathrm{Cu} \mathrm{K \alpha}$ radiation $(\lambda=1.54056 \AA)$, and the data was acquired in the range of $10-60^{\circ}(2 \theta)$ at a rate of $5^{\circ} \mathrm{min}^{-1}$. Ultraviolet-visible diffuse reflectance spectra were performed for the dry-pressed disk samples with an UV-vis spectrophotometer (U-3010, Hitachi) using $\mathrm{BaSO}_{4}$ as the reflectance standard. Fourier transform infrared spectroscope (FTIR) spectra were collected on a Nicolet-560 spectrometer. Atomic force microscopy (AFM) measurement of the a- $\mathrm{TiO}_{2}$ coating on silicon wafer was performed under tapping mode in a closed fluid cell filled with deionized water using BRUKER Dimension Icon. Photoluminescence (PL) spectra was recorded on Jobin Yvon Fluorolog 3-21 fluorescence spectrometer with excitation at $350 \mathrm{~nm}$. Time-resolved transient PL decay curves were obtained on 
Jobin Yvon Fluorolog 3-21 fluorescence spectrometer under the excitation of $390 \mathrm{~nm}$ and probed at $460 \mathrm{~nm}$. Transient absorption (TA) spectroscopy was performed on Edinburgh LP980-KS transient absorption spectrometer equip with a Nd:YAG flash pump laser (an excitation source of $355 \mathrm{~nm}$ laser with $\sim 7 \mathrm{~ns}$ pulse width) and an CCD camera array detector.

\section{Results and Discussion}

\section{Preparation and Characterizations of GCN@a-TiO 2 Core@Shell Photocatalyst}
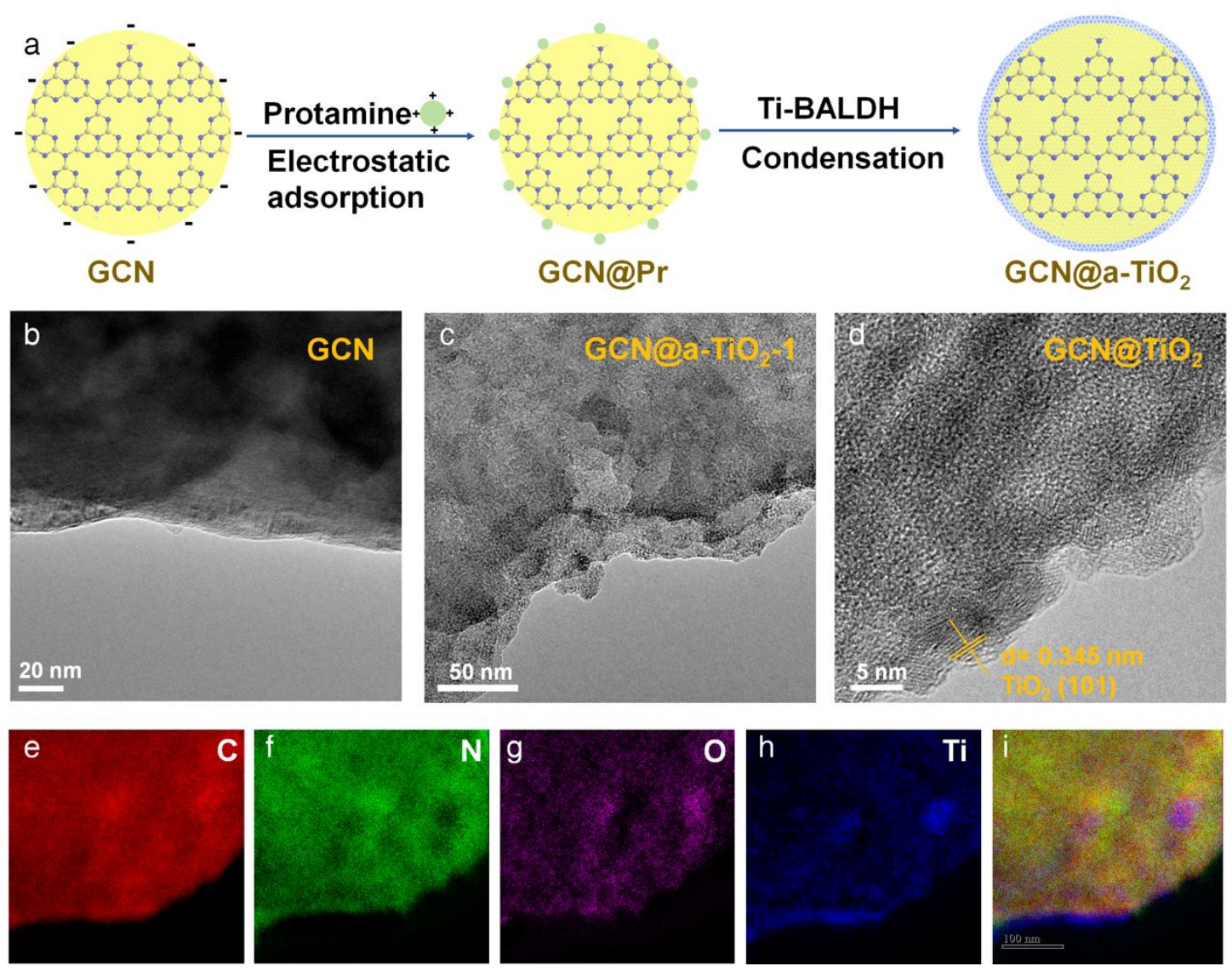

Figure 1. (a) Schematic preparation of GCN@a-TiO 2 core@shell photocatalyst. (b-d)

High-resolution TEM images of (b) GCN, (c) GCN@a-TiO $2-1$ and (d) GCN@TiO $2-1$ calcined under $480^{\circ} \mathrm{C}$ in air for $2 \mathrm{~h}$. (e-i) Electron energy loss spectroscopy (EELS) elemental mappings of GCN@a-TiO $2-1$. Red for carbon; green for nitrogen; purple for oxygen; blue for titanium. (i) The merged image of (e-h). 
GCN@a-TiO 2 core@shell photocatalyst was prepared through protamine-induced mineralization of amorphous titania $\left(\mathrm{a}-\mathrm{TiO}_{2}\right)$ on graphic carbon nitrate $(\mathrm{GCN})$ under room temperature in aqueous solution (Figure 1a). Specifically, positive charged protamine molecules were first adsorbed on the negatively charged surface of GCN through electrostatic interaction. ${ }^{29}$ The absorbed protamine then catalyzed the hydrolysis and condensation of titanium (IV) bis (ammonium lactato) dihydroxide (Ti-BALDH) and in situ deposition of a-TiO 2 nanoshell on GCN (GCN@a-TiO $2-1) .{ }^{24}$ To validate the deposition of the $\mathrm{a}-\mathrm{TiO}_{2}$ nanoshell, the topological and chemical structures of GCN@a-TiO 2 -1 were examined by high-resolution transmission electron microscopy (HR-TEM) and electron energy loss spectroscopy (EELS). Compared with GCN (Figure 1b), GCN@a-TiO -1 exhibited similar topological structure (Figure 1c), indicating amorphous nature of the mineralized titania. Subsequently, GCN@a- $\mathrm{TiO}_{2}-1$ was calcined under $480{ }^{\circ} \mathrm{C}$ in air to examine the crystalline structure of deposited titania. As shown in Figure 1d, the crystal lattice corresponding to the (101) plane of anatase became rather apparent after calcination, verifying the successful deposition of amorphous titania on GCN. Moreover, EELS mappings reveled the well distribution of all elements, including O, Ti, C and N (Figure 1e-i). Amongst, $\mathrm{C}$ and $\mathrm{N}$ elements should be originated from GCN, while $\mathrm{O}$ and Ti elements should be assigned to a- $\mathrm{TiO}_{2}$. This indicated a- $\mathrm{TiO}_{2}$ was deposited on the entire surface of GCN. 

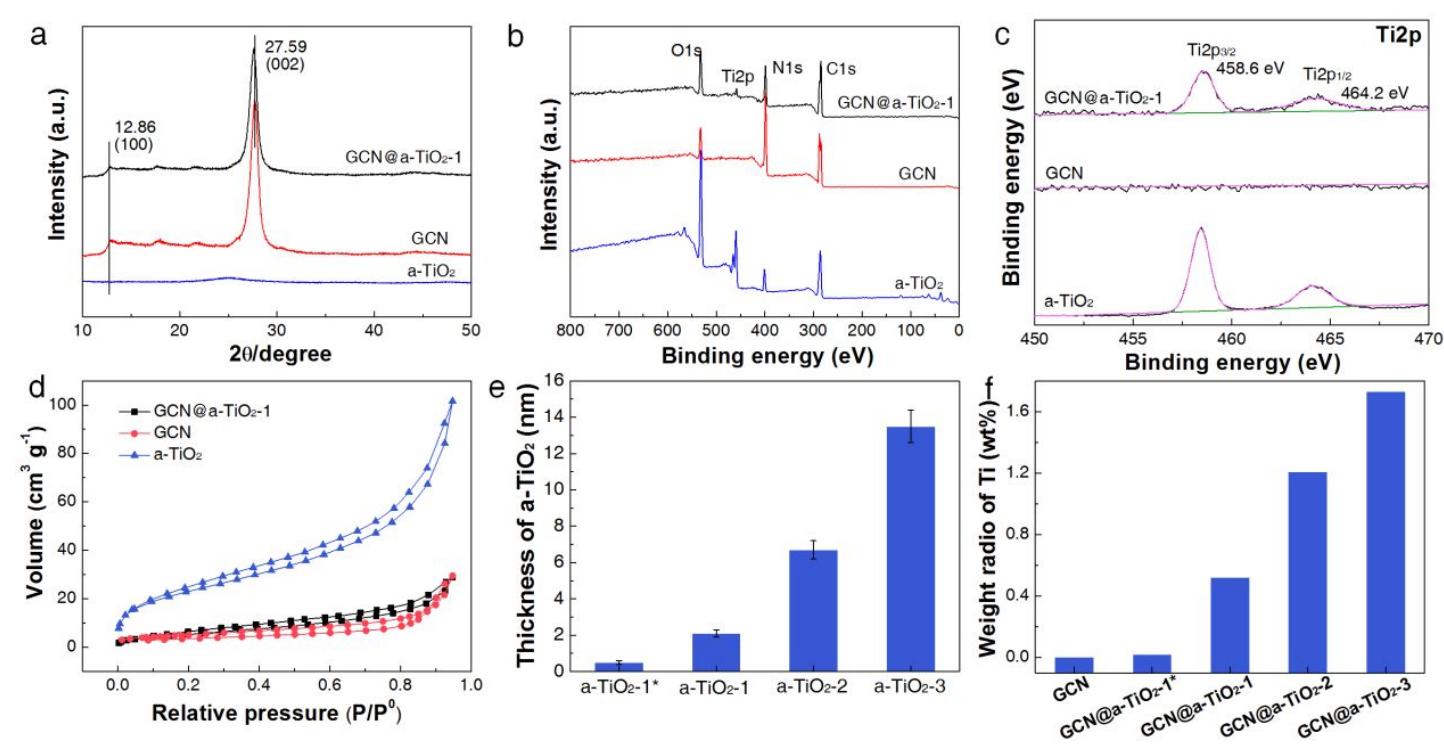

Figure 2. (a) XRD patterns and (b) XPS spectra of GCN@a- $\mathrm{TiO}_{2}-1, \mathrm{GCN}$ and $\mathrm{a}-\mathrm{TiO}_{2}$.

(c) High-resolution Ti 2p XPS spectra of GCN@a-TiO $-1, \mathrm{GCN}$ and $\mathrm{a}-\mathrm{TiO}_{2}$. (d) $\mathrm{N}_{2}$ adsorption-desorption isotherms of $\mathrm{GCN} @ \mathrm{a}-\mathrm{TiO}_{2}-1, \mathrm{GCN}$ and $\mathrm{a}-\mathrm{TiO}_{2}$. (e) The thickness changes of a- $\mathrm{TiO}_{2}$ coating on silicon wafer measured by AFM. (f) The weight ratio of $\mathrm{Ti}$ element of $\mathrm{GCN}, \mathrm{GCN} @ \mathrm{a}-\mathrm{TiO}_{2}-1 *\left(0.5 \mathrm{mg} \mathrm{mL} \mathrm{m}^{-1}\right.$ protamine), GCN@a-TiO $2-1$, GCN@a-TiO $2-2, \mathrm{GCN} @ \mathrm{a}-\mathrm{TiO}_{2}-3$ and $\mathrm{a}-\mathrm{TiO}_{2}$ calculated from the EDS results.

The existence and content of $\mathrm{a}-\mathrm{TiO}_{2}$ on $\mathrm{GCN}$ were further determined by X-ray powder diffraction (XRD), Fourier transform infrared spectroscope (FTIR) and X-ray photoelectron spectroscopy (XPS). As shown in Figure 2a, GCN@a-TiO ${ }_{2}-1$ exhibited similar peaks with GCN at $12.86^{\circ}$ (100) and $27.59^{\circ}$ (002), which corresponded to the in-planar packing of tri-s-triazine and the interlayer stacking of the conjugated aromatic system. ${ }^{30}$ The interlayer distance was calculated to be 0.323 $\mathrm{nm}$, indicating the negligible influence of the mineralization process on the GCN 
structure, in line with the FTIR results (Figure S1). None of other diffraction peak representing titania was found, again evidencing the amorphous nature of a- $\mathrm{TiO}_{2}$ nanoshell. The elemental composition of GCN@a-TiO $2-1, \mathrm{GCN}$ and $\mathrm{a}-\mathrm{TiO}_{2}$ was then characterized by XPS. As shown in Figure 2b, GCN@a-TiO ${ }_{2}-1$ exhibited typical peaks of $\mathrm{C}, \mathrm{N}, \mathrm{O}$ and Ti elements. In deconvoluted Ti2p spectra, two characteristic peaks corresponding to Ti2p3/2 and Ti2p1/2 at 458.6 and $464.2 \mathrm{eV}$ were observed (Figure 2c). ${ }^{24}$ High-resolution C1s XPS spectra (Figure S2a) could be deconvoluted into three peaks at $284.81,286.23$ and $288.19 \mathrm{eV}$. Two main peaks at 284.81 and $288.19 \mathrm{eV}$ were assigned to $\mathrm{C}-\mathrm{C}$ bond and $\mathrm{N}-\mathrm{C}=\mathrm{N}$ bond of $\mathrm{GCN}$, respectively. The peak of $286.23 \mathrm{eV}$ corresponded to $\mathrm{C}-\mathrm{O}$ bond of protamine and GCN calcined in air. ${ }^{15}$ For N1s spectra, the peaks were assigned to C-N-H at $\sim 400.92 \mathrm{eV}, \mathrm{C}-(\mathrm{N})_{3}$ at $\sim 399.81$ $\mathrm{eV}$ and $\mathrm{C}-\mathrm{N}=\mathrm{C}$ at $\sim 398.53 \mathrm{eV}$, respectively (Figure $\mathbf{S 2 b}$ ). ${ }^{31}$ The texture structure examined by $\mathrm{N}_{2}$ adsorption-desorption isotherms (Figure 2d and Table S1) showed that GCN@a-TiO $2-1\left(20.9 \mathrm{~m}^{2} \mathrm{~g}^{-1}\right)$ possessed higher surface area than GCN $\left(13.9 \mathrm{~m}^{2}\right.$ $\left.\mathrm{g}^{-1}\right)$, which was probably due to the deposition of a- $\mathrm{TiO}_{2}$ nanoshell (a-TiO, $84.2 \mathrm{~m}^{2}$ $\left.\mathrm{g}^{-1}\right)$. Similar surface area, pore volume and pore size distribution of GCN@a-TiO $2-1$, GCN@a-TiO $2-2$ and $\mathrm{GCN} @ a-\mathrm{TiO}_{2}-3$ suggested that the increase of mineralization cycles may only thicken the a- $\mathrm{TiO}_{2}$ nanoshell rather than interfering its internal structure (Table S1 and Figure S3). Since the nanoshell structure of a core@shell photocatalyst would have great impact on both behaviors of electron transfer and molecule diffusion during the photocatalytic reaction, the thickness of the a- $\mathrm{TiO}_{2}$ nanoshell was regulated by changing the concentration of protamine and the 
mineralization cycles. To reveal the thickness changes of $\mathrm{a}-\mathrm{TiO}_{2}$ nanoshell prepared under different conditions, silicon wafer was used instead of GCN as substrate for a- $\mathrm{TiO}_{2}$ deposition, and atomic force microscopy (AFM) was employed to measure the thickness of a- $\mathrm{TiO}_{2}$ coating. As shown in Figure $\mathbf{S 4 a}$, the a- $\mathrm{TiO}_{2}-1$ coating on silicon wafer was generally continuous and compact, which showed the thickness of $2.1 \pm 0.2$ nm obtained by measuring the height change of silicon wafer with and without a- $\mathrm{TiO}_{2}$ (Figure S4b). Using the same method, the thickness of a-TiO ${ }_{2}$ deposited under different conditions was acquired (Figure 2e), which increased gradually with the increase of protamine concentration and mineralization cycle, in line with the previous literature. ${ }^{24}$ The weight ratio of Ti on the surface region of GCN@a-TiO 2 got increased from $0.02 \%$ to $1.73 \%$ with the increase of protamine concentration and mineralization cycle as indicated by the EDS results (Figure 2f).
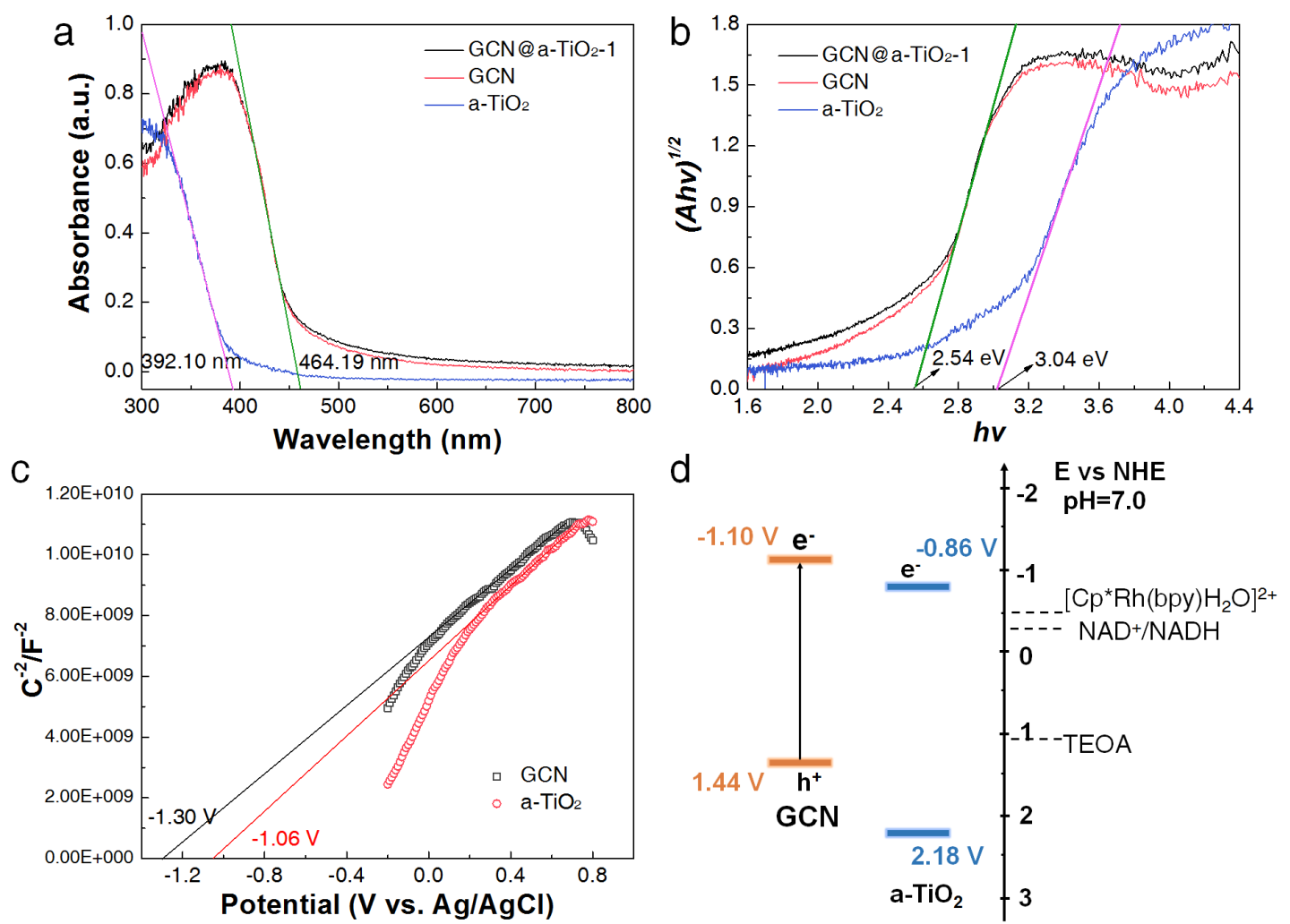
Figure 3. (a) UV-Vis diffuse reflectance spectra and (b) the corresponding tauc plots of GCN@a-TiO $2-1$, GCN and a-TiO 2 . (c) Mott-Schottky plot for GCN and a-TiO electrodes measured in $0.1 \mathrm{~mol} \mathrm{~L}-1 \mathrm{Na}_{2} \mathrm{SO}_{4}$ solution at $1 \mathrm{kHz}$ in the dark. (d) Schematic band structure of $\mathrm{GCN}$ and $\mathrm{a}-\mathrm{TiO}_{2}$, and the redox potential of $\left[\mathrm{Cp} * \mathrm{Rh}(\text { bpy }) \mathrm{H}_{2} \mathrm{O}\right]^{2+}, \mathrm{NAD}^{+}$and TEOA.

The band structure of GCN and a-TiO ${ }_{2}$ in $\mathrm{GCN} @ \mathrm{a}-\mathrm{TiO}_{2}$ was then characterized based on several techniques, including UV-Vis diffuse reflection spectroscopy (DRS), XPS valence band (VB) spectra and Mott-Schottky plot. As shown in Figure 3a, GCN@a-TiO $2-1$ and GCN exhibited the same absorption edge of 464.19 nm, suggesting that the deposition of $\mathrm{a}-\mathrm{TiO}_{2}$ did not have impact on the light absorption of GCN. GCN and GCN@a-TiO 2 exhibited similar bandgap of $\sim 2.54$ eV from the tauc plots (Figure 3b), while a- $\mathrm{TiO}_{2}$ showed a higher bandgap of $3.04 \mathrm{eV}$, which allowed visible light transmission through the a- $\mathrm{TiO}_{2}$ nanoshell and did not affect the visible light absorption of GCN. ${ }^{32}$ Mott-Schottky analysis (Figure 3c) further indicated the flat-band potential of $\mathrm{GCN}$ and $\mathrm{a}-\mathrm{TiO}_{2}$ being located at -1.10 and $-0.86 \mathrm{~V}$ (vs NHE, $\mathrm{pH}=7.0$ ), which could be viewed as the $\mathrm{CB}$ values of $\mathrm{GCN}$ and $\mathrm{a}-\mathrm{TiO}_{2}$, respectively. ${ }^{33}$ Combined with the analysis of bandgap derived from the DRS spectra, the valence bands (VB) potentials of $\mathrm{GCN}$ and $\mathrm{a}-\mathrm{TiO}_{2}$ were calculated to be 1.44 and $2.18 \mathrm{~V}$ ( $v \mathrm{~s}$ NHE, $\mathrm{pH}=7.0$ ), respectively (Figure 3d). The CB offset of $0.24 \mathrm{~V}$ between GCN and a- $\mathrm{TiO}_{2}$ may facilitate the transfer of electrons from $\mathrm{GCN}$ to $\mathrm{a}-\mathrm{TiO}_{2}$.

All above results indicated the formation of porous, conductive a- $\mathrm{TiO}_{2}$ nanoshell 
with controllable thickness on the surface of GCN. Besides, such a- $\mathrm{TiO}_{2}$ nanoshell could be further deposited on other semiconductor cores, such as $\mathrm{CdS}, \mathrm{ZnO}$ and $\mathrm{SrTiO}_{3}$, (Figure S6 and S7) by other organic inducers, such as PAH, lysozyme and arginine (Figure S6). Therefore, the semiconductor@a-TiO $\mathrm{T}_{2}$ core@shell photocatalyst prepared through bioinspired mineralization could set a good sample to judge the coordinated optimization between photo-generated electron transfer and electron donor molecule diffusion.

\section{Coordination between Photo-Generated Electron Transfer and Electron Donor}

\section{Molecule Diffusion through a-TiO ${ }_{2}$ Nanoshell}
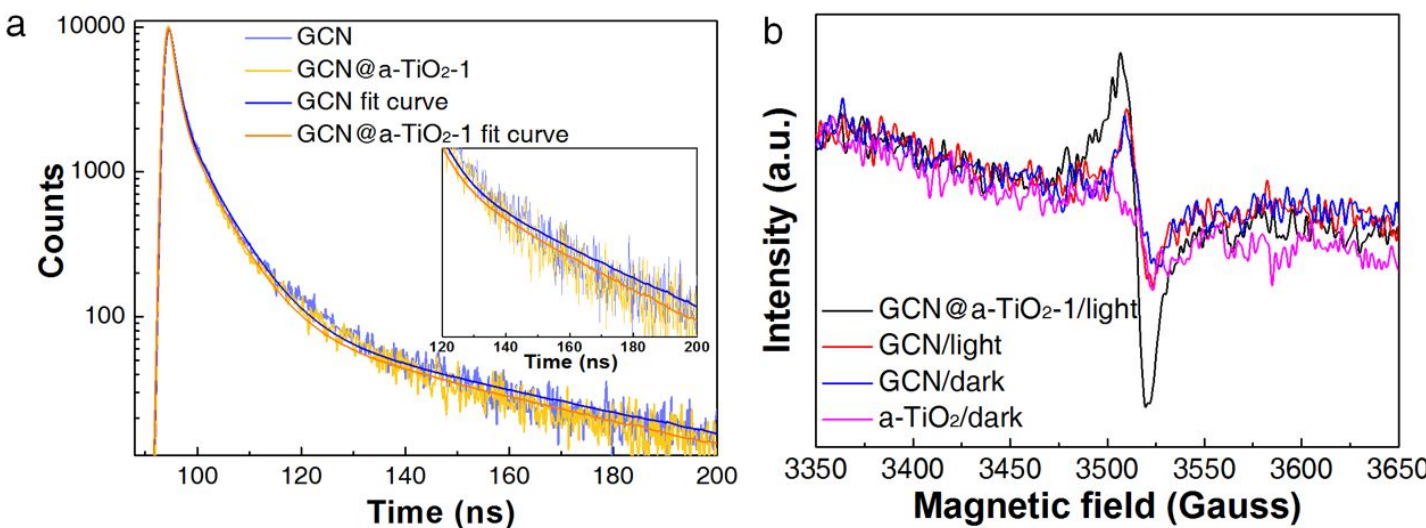

C

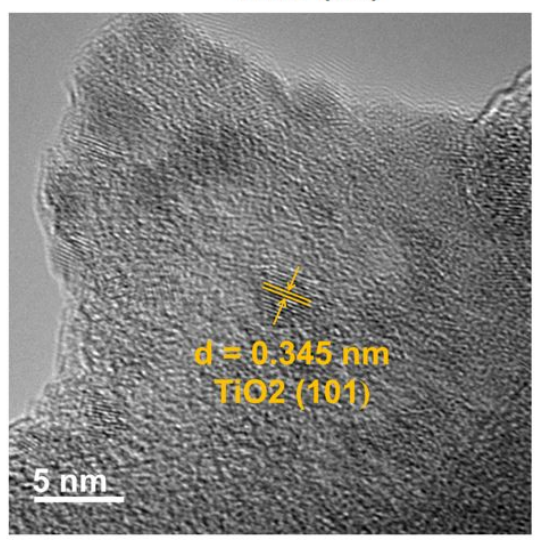

d

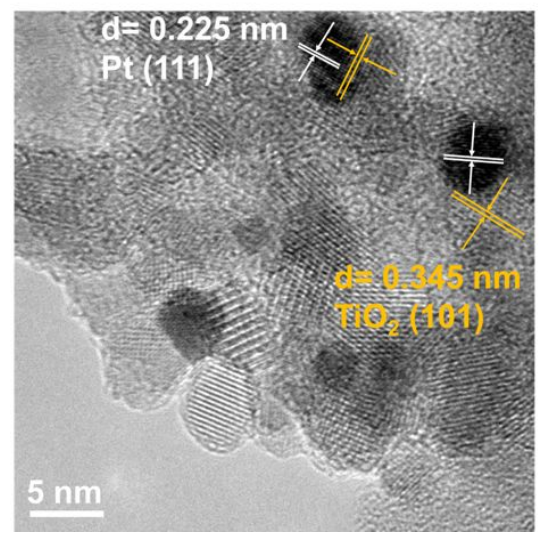

Figure 4. (a) Time-resolved transient PL spectra of GCN@a-TiO $\mathrm{a}_{2}-1$ and GCN. (b)

EPR spectra of GCN@a- $\mathrm{TiO}_{2}, \mathrm{GCN}$ and $\mathrm{a}-\mathrm{TiO}_{2}$ under light and dark condition. (c-d)

HRTEM images of $\mathrm{GCN} @ \mathrm{a}-\mathrm{TiO}_{2}$ treated by $\mathrm{H}_{2} \mathrm{PtCl}_{6}$ under (c) dark and (d) light 
conditions followed by heating under $480{ }^{\circ} \mathrm{C}$ in air to crystallize the titania.

Since it was the first time to adopt bioinspired a- $\mathrm{TiO}_{2}$ nanoshell to coordinate the photo-generated electron transfer and the electron donor molecule diffusion, the electron transfer behavior of the $\mathrm{a}-\mathrm{TiO}_{2}$ nanoshell on the $\mathrm{GCN}$ core should be in-depth elucidated. As shown in the steady-state PL (Figure S8), all samples of GCN@a-TiO 2 exhibited weakened luminescence intensities by contrasting with GCN. This indicated $\mathrm{a}-\mathrm{TiO}_{2}$ in the nanoshell could extract electrons from GCN. ${ }^{34,35}$ Then, time-resolved transient PL was conducted to show the electron transfer behavior through the a- $\mathrm{TiO}_{2}$ nanoshell. As shown in Figure 4a, GCN@a-TiO ${ }_{2}-1$ exhibited a shorter life time than GCN (30.7 vs $33.8 \mathrm{~ns}$ ), further evidencing the electron transfer from $\mathrm{GCN}$ to $\mathrm{a}^{-\mathrm{TiO}_{2}}$ (Table S2). ${ }^{36}$ Decreased steady-state photoluminescence intensity and intensity-average lifetime $\left(\tau_{\mathrm{A}}\right)$ of the emission decay further suggested that electron-hole recombination was suppressed by the rapid electron transfer from GCN to a- $\mathrm{TiO}_{2} \cdot{ }^{37}$ Since the trap states at the GCN surface would also cause shorter PL lifetimes, EPR spectra of GCN@a-TiO $2-1, \mathrm{GCN}$ and $\mathrm{a}-\mathrm{TiO}_{2}$ under light and dark condition were further performed to validate the electron transfer process. As shown in Figure 4b, GCN exhibited a weak Lorentzian line, which corresponded to the unpaired electrons on the polymeric heptazine rings of GCN. ${ }^{38}$ After illuminated by a $300 \mathrm{~W}$ xenon lamp ( $\left.\geq 420 \mathrm{~nm}, 100 \mathrm{~mW} \mathrm{~cm}^{-2}\right)$, minor changes were observed for GCN, while a stronger EPR signal was observed for $\mathrm{GCN} @ \mathrm{a}-\mathrm{TiO}_{2}$. This should be arisen from the formation of $\mathrm{Ti}^{3+}$ due to the electron transfer from $\mathrm{GCN}$ to $\mathrm{a}-\mathrm{TiO}_{2}$. It was 
also reported $\mathrm{Pt}^{4+}$ could be reduced into metallic $\mathrm{Pt}^{0}$ and further deposited on the position bearing enriched photo-generated electrons for a photocatalyst. ${ }^{39}$ The position could reflect the electron transfer direction during photocatlytic reaction. In this context, photochemical reduction of $\mathrm{Pt}^{4+}$ was conducted by GCN@a-TiO $\mathrm{T}_{2}$ under visible light (AM 1.5G). To identify the deposition position of metallic $\mathrm{Pt}^{0}$, the as-synthesized Pt@GCN@a-TiO ${ }_{2}$ was calcined in air to crystallize the amorphous titania. For comparison, GCN@a- $\mathrm{TiO}_{2}$ was also treated by $\mathrm{Pt}^{4+}$ under dark condtion, followed by water washing and calcination in air (Figure 4c). By contrast with Figure 4c, Figure 4d showed the typical crystal lattice of Pt (111) on the top of the crystal lattice of titania (101), validating the electron enrichment on a- $\mathrm{TiO}_{2}$ and the oriented electron transfer from $\mathrm{GCN}$ to $\mathrm{a}-\mathrm{TiO}_{2}$. 


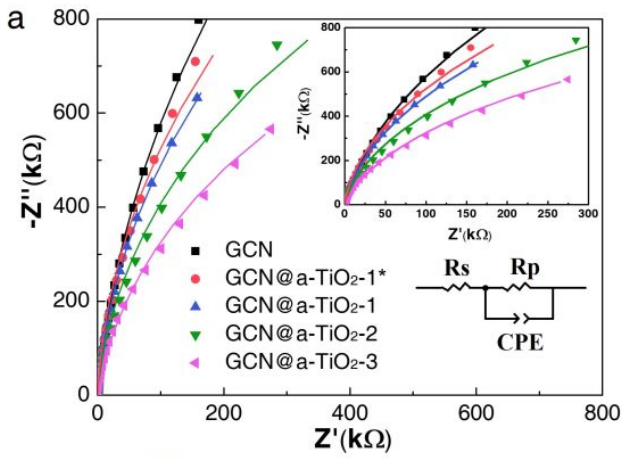

b

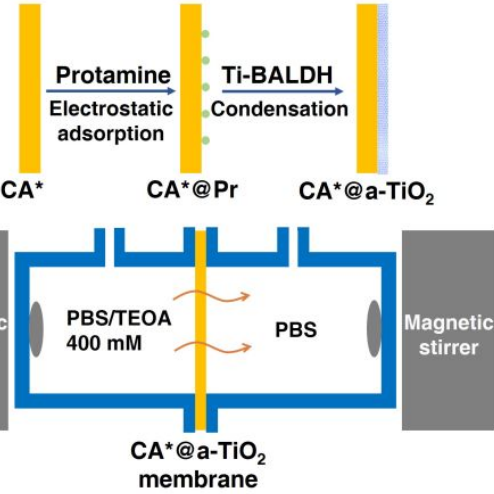

d

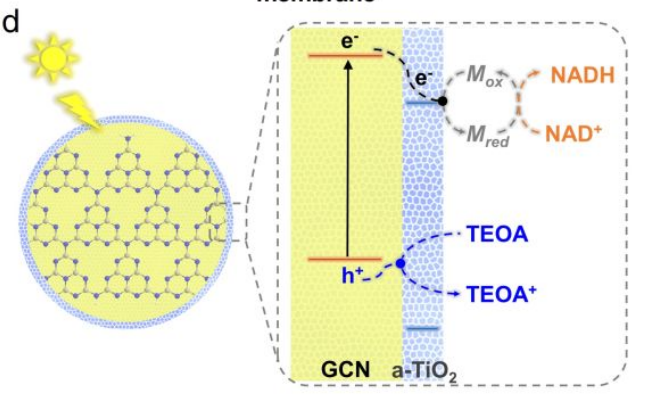

$\mathrm{C}$

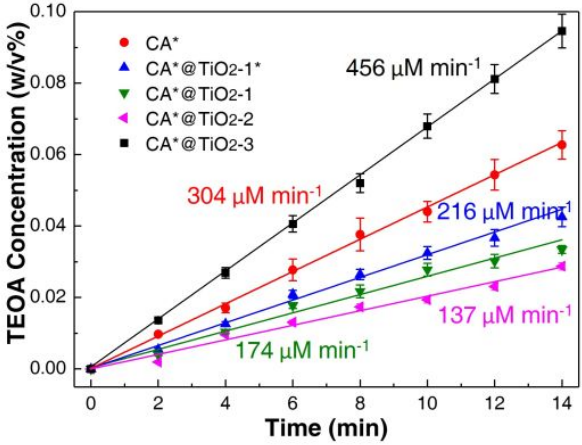

e

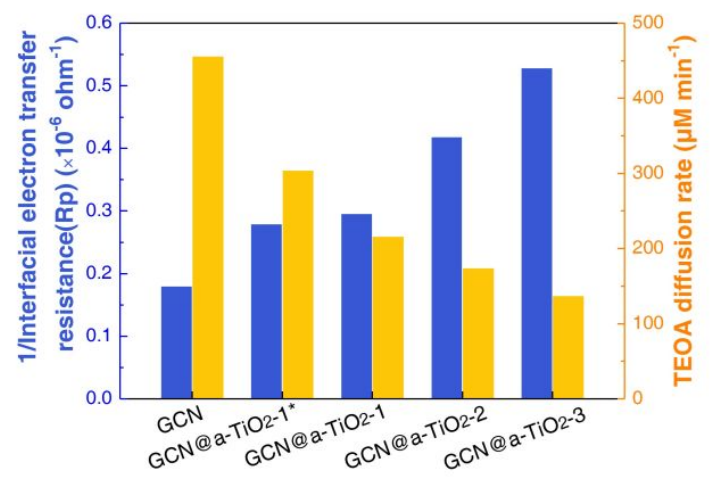

Figure 5. (a) EIS Nyquist plots. (b) Schematic preparation of $\mathrm{CA} * @ a-\mathrm{TiO}_{2}$ membranes and a home-made diffusion device. (c) Diffusion rate of TEOA through $\mathrm{CA} * \mathrm{a}-\mathrm{TiO}_{2}$ membranes. Note that CA referred to cellulose acetate membrane, which was pre-treated with dopamine $\left(2 \mathrm{mg} \mathrm{mL}^{-1}\right)$ tris- $\mathrm{HCl}$ solution for $2 \mathrm{~h}$ to enhance the surface hydrophilicity and narrow the pore size. The pre-treated CA membrane was denoted as $\mathrm{CA}^{*}$ membrane. (d) Schematic interfacial electron transfer and TEOA diffusion through the nanoshell of $\mathrm{GCN} @ \mathrm{a}-\mathrm{TiO}_{2}$ photocatalyst. (e) Interfacial electron transfer resistance of GCN, GCN@a-TiO ${ }_{2}-1^{*}, \mathrm{GCN} @ \mathrm{a}-\mathrm{TiO}_{2}-1$, 
GCN@a-TiO 2 -2 and GCN@a-TiO $2-3$, as well as TEOA diffusion rate of CA*, $\mathrm{CA} * \mathrm{a}-\mathrm{TiO}_{2}-1 *, \mathrm{CA} * @ \mathrm{a}-\mathrm{TiO}_{2}-1, \mathrm{CA}^{*} @ \mathrm{a}-\mathrm{TiO}_{2}-2$ and $\mathrm{CA} * @ \mathrm{a}-\mathrm{TiO}_{2}-3$.

The coordination between electron transfer and molecule diffusion through the a- $\mathrm{TiO}_{2}$ nanoshell was then elucidated by altering the nanoshell thickness. The interfacial electron transfer resistance was evaluated by EIS Nyquist plots. As shown in Figure 5a, with the increase of a- $\mathrm{TiO}_{2}$ nanoshell thickness, the radius of the curve decreased, suggesting the lower interfacial electron transfer resistance for thicker a- $\mathrm{TiO}_{2}$ nanoshell. The interfacial electron transfer resistance could be calculated through the equivalent circuit (Figure 5a), where Rp corresponded to the interfacial electron transfer resistance. ${ }^{28}$ According to the fitting results, Rp of GCN, GCN@a-TiO $2-1 *, \quad G C N @ a-\mathrm{TiO}_{2}-1, \mathrm{GCN} @ \mathrm{a}-\mathrm{TiO}_{2}-2$ and $\mathrm{GCN} @ \mathrm{a}-\mathrm{TiO}_{2}-3$ were calculated to be $5.55 \times 10^{6}, 3.58 \times 10^{6}, 3.38 \times 10^{6}, 2.39 \times 10^{6}$ and $1.89 \times 10^{6} \mathrm{ohm}$, respectively.GCN@a-TiO $2-3$ exhibited the smallest semi-diameter and the lowest Rp value, in line with the results from steady-state PL spectra (Figure S8). This further demonstrated that thicker a- $\mathrm{TiO}_{2}$ nanoshell could decrease the interfacial electron transfer resistance and promote charge separation. The more efficient electron transfer for thicker a- $\mathrm{TiO}_{2}$ nanoshell should be arisen from the larger interfacial area between GCN and $\mathrm{a}-\mathrm{TiO}_{2}$, and the inhibited charge recombination by separating electrons and holes over a larger distance.

In addition to extracting electrons from $\mathrm{GCN}$, the a- $\mathrm{TiO}_{2}$ nanoshell should also allow the free diffusion of TEOA to consume the photo-induced holes on the surface 
of GCN. As shown in Figure S3, the pore diameter of a- $\mathrm{TiO}_{2}$ nanoshell was $\sim 3.3 \mathrm{~nm}$, which was $\sim 5$ times larger than TEOA $\left(0.59 \times 0.61 \times 0.63 \mathrm{~nm}^{3}\right)$. Hence, TEOA could diffuse through a- $\mathrm{TiO}_{2}$ nanoshell to the surface of GCN. To prove the diffusion of TEOA, we designed a home-made device with a- $\mathrm{TiO}_{2}$ nanolayer coated on pre-treated cellulose acetate (denoted as $\mathrm{CA}^{*}$ ) membrane as the support (Figure 5b). For CA*, the TEOA diffusion rate was $456 \mu \mathrm{M} \min ^{-1}$ (Figure 5c). After coating one layer of a- $\mathrm{TiO}_{2}$ under $0.5 \mathrm{mg} \mathrm{mL} \mathrm{m}^{-1}$ protamine (to mimic $\mathrm{GCN} @ \mathrm{a}-\mathrm{TiO}_{2}-1^{*}$ ), the TEOA diffusion rate decreased to $304 \mu \mathrm{M} \mathrm{min}{ }^{-1}$. This suggested that a- $\mathrm{TiO}_{2}$ nanoshell exerted diffusion resistance to TEOA but still allow its penetration. With the increase of a-TiO ${ }_{2}$ thickness on $\mathrm{CA}^{*}$ membrane to mimic GCN@a-TiO $-1, \mathrm{GCN} @ \mathrm{a}-\mathrm{TiO}_{2}-2$ and GCN@a-TiO $2-3$, the TEOA diffusion rate further decreased to 216, 174 and 137 $\mu \mathrm{M} \min ^{-1}$. This suggested that although thicker a- $\mathrm{TiO}_{2}$ nanoshell reduced the electron transfer resistance, it also increased the molecule diffusion resistance (Figure 5d and 5e). The performance of GCN@a-TiO 2 core@shell photocatalyst should be governed by both behaviors of electron transfer and molecule (TEOA) diffusion. Thus, an optimal nanoshell thickness exists toward the highest photocatalytic efficiency.

\section{Photocatalytic Nicotinamide Regeneration.}




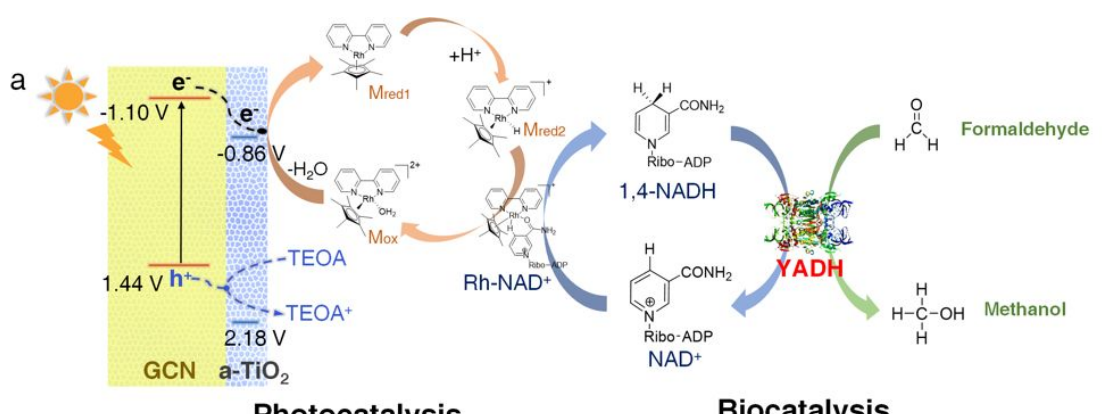

Photocatalysis

Biocatalysis
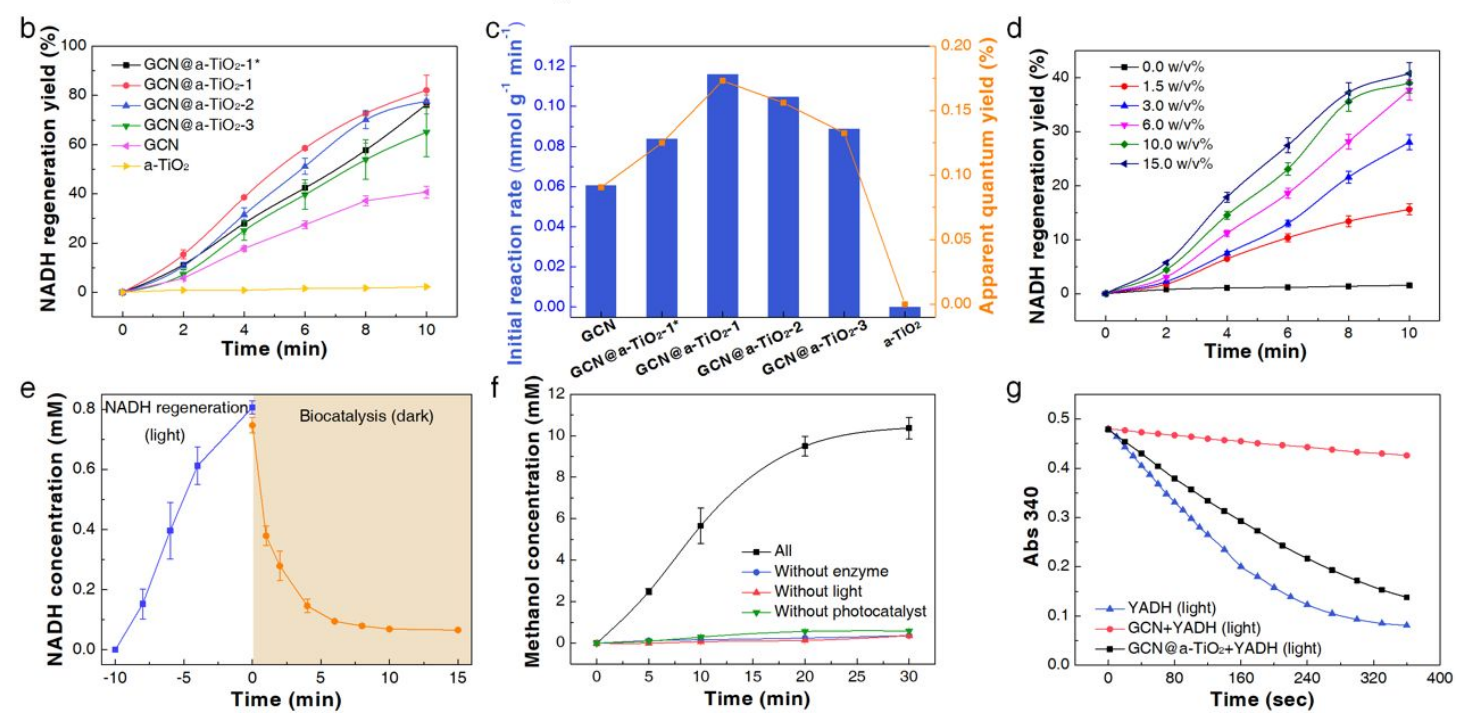

Figure 6. (a) Schematic photocatalytic NADH regeneration and photo-bio-coupled catalytic conversion of formaldehyde into methanol. (b) Photocatalytic NADH regeneration by $\mathrm{GCN} @ \mathrm{a}-\mathrm{TiO}_{2}, \mathrm{GCN}$ and $\mathrm{a}-\mathrm{TiO}_{2}$, and (c) the corresponding initial reaction rate and apparent quantum yield (2-4 min). (d) Photocatalytic NADH regeneration by GCN with different TEOA concentration. (e) NADH regeneration and enzymatic degradation enabled by $\mathrm{GCN} @ \mathrm{a}^{-\mathrm{TiO}_{2}-1}$ and YADH.

Photo-bio-coupled catalytic production of methanol. (g) The activity of YADH after incubated with GCN and GCN@a-TiO 2 for 1 h under LED light illumination $(\lambda=405$ $\mathrm{nm})$.

In the photo-bio-coupled catalytic system, the expensive nicotinamide cofactor NADH activates oxidoreductase and provided hydrides for biocatalytic reaction. 
Therefore, efficient and selective regeneration of NADH is an important process for assessing photo-bio-coupled catalytic system. ${ }^{40-41}$ Based on the above investigation, the NADH regeneration performance was evaluated by utilizing $\left[\mathrm{Cp} * \mathrm{Rh}(\mathrm{bpy}) \mathrm{H}_{2} \mathrm{O}\right]^{2+}$ as an electron mediator (M) and TEOA as a hole scavenger (also called electron donor). ${ }^{25,42}$ Upon the absorption of visible light by GCN, electrons would be excited to the $\mathrm{CB}$ of $\mathrm{GCN}$. The electrons then transferred to the $\mathrm{CB}$ of $\mathrm{a}-\mathrm{TiO}_{2}$ nanoshell. The holes left on the GCN surface were consumed by TEOA that diffused through the a- $\mathrm{TiO}_{2}$ nanoshell. Finally, $\mathrm{M}$ accepted the electrons, accomplishing the selective reduction of $\mathrm{NAD}^{+}$(Figure 6a). Notably, the regenerated NADH was detected by measuring its characteristic absorption at $340 \mathrm{~nm}$ and confirmed by enzymatic conversion (Figure S9). And, we could confirm that the oxidized product of TEOA have little impact on the reaction system (Figure S10).

As shown in Figure 6b and 6c, all samples of GCN@a-TiO ${ }_{2}$ exhibited higher NADH regeneration efficiency than GCN, while the NADH regeneration efficiency exerted a volcano-shape curve as the a- $\mathrm{TiO}_{2}$ nanoshell thickness increased. The phenomenon of nanoshell thickness-dominated NADH regeneration efficiency should be arisen from the competing between electron transfer from $\mathrm{GCN}$ to $\mathrm{a}-\mathrm{TiO}_{2}$ and diffusion of TEOA through a- $\mathrm{TiO}_{2}$ nanoshell to consume holes. It should be noted that the influence of electron transfer efficiency from the photocatalyst to $M$ was excluded through using an excessive amount of M (Figure S11). As demonstrated in previous part, thicker a- $\mathrm{TiO}_{2}$ nanoshell exhibited higher electron transfer rate but larger TEOA diffusion resistance. For GCN, GCN@a-TiO ${ }_{2}-1 *$ and $\mathrm{GCN} @ \mathrm{a}-\mathrm{TiO}_{2}-1$, 
the $\mathrm{a}-\mathrm{TiO}_{2}$ nanoshell thickness was increased, the initial NADH regeneration rate and apparent quantum yield increased from 0.06 to $0.12 \mathrm{mmol} \mathrm{g}^{-1} \mathrm{~min}^{-1}$ and $0.09 \%$ to $0.17 \%$, respectively, due to the dominant function of facilitated electron transfer arisen from the a-TiO 2 . Further increasing the nanoshell thickness, the initial NADH regeneration rate and apparent quantum yield decreased from 0.12 to $0.09 \mathrm{mmol} \mathrm{g}^{-1}$ $\min ^{-1}$ and $0.17 \%$ to $0.13 \%$, which was probably owing to larger TEOA diffusion resistance. The increase of TEOA diffusion resistance would inhibit the diffusion of TEOA through the nanoshell and further reduced the TEOA concentration near the surface of GCN. The lower TEOA concentration would decrease the consumption rate of the photo-generated holes and then increase the possibility of electron-hole recombination, thus lowering the $\mathrm{NADH}$ regeneration rate. The assumption was further validated by examining the effect of TEOA concentration on the NADH regeneration rate of $\mathrm{GCN}$, where the $\mathrm{NADH}$ regeneration rate got increased with the increase of TEOA concentration on the surface of GCN (Figure 6d). As a result, after coordination between the electron transfer and TEOA diffusion through regulating the a- $\mathrm{TiO}_{2}$ nanoshell thickness, GCN@a-TiO $\mathrm{T}_{2}-1$ exhibited the highest NADH regeneration yield of $\sim 82.1 \%$ after reaction equilibrium, which also exerted the highest initial reaction rate $\left(\sim 0.12 \mathrm{mmol} \mathrm{g}^{-1} \mathrm{~min}^{-1}\right)$ and apparent quantum efficiency $(\sim 0.17 \%)$ (Figure 6c). Importantly, the optimized NADH regeneration yield was over 200\% higher than GCN. Meanwhile, GCN@a-TiO $2-1$ photocatalyst also exerted excellent operational stability and retained its original structure after the photocatalytic NADH regeneration (Figure S12). As a matter of fact, during the 
photocatalytic NADH regeneration, the photo-generated charge carriers (electrons and holes) were probably involved in the oxidation of water and TEOA by holes and the reduction of oxygen by electrons to form reactive oxygen species (ROS), which may destroy the structure of NADH. Detailed discussion about the generated ROS $(\cdot \mathrm{OH}$, $\cdot \mathrm{O}_{2}{ }^{-}$and other radicals), and their influence on the structure of NADH were presented in the supporting information (Page S3 and Figure S13).

To evaluate the enzymatic activity of regenerated NADH, yeast alcohol dehydrogenase (YADH) was coupled with $\mathrm{GCN} @ \mathrm{a}-\mathrm{TiO}_{2}$ for photo-bio-coupled catalytic hydrogenation of formaldehyde to methanol. ${ }^{15,43}$ First, photocatalytic NADH regeneration and enzymatic hydrogenation were conducted successively. As shown in Figure 6e, NADH was successfully accumulated during the visible light illumination. After adding YADH and formaldehyde in the regeneration solution, NADH was completely oxidized in 10 min (Figure S15), indicating enzymatically active feature of the regenerated NADH. Moreover, the methanol product was measured under continuous visible light illumination. As shown in Figure 6f, the concentration of methanol got gradually increased and $\sim 10.4 \mathrm{mM}$ methanol was detected after $30 \mathrm{~min}$ illumination. By contrast, no methanol was detected in the absence of light, YADH or photocatalyst, verifying the reaction scheme in Figure 6a.

The compatibility of photocatalyst and enzyme is crucial when the photocatalytic NADH regeneration is coupled with the enzymatic hydrogenation of formaldehyde by YADH. During the light illumination, GCN and YADH were incubated for $1 \mathrm{~h}$, and YADH was almost completely inactivated (Figure 6g). We found that the 
photo-generated holes of GCN damaged the active site and structure of YADH. The active site of $\mathrm{YADH}$ is formed by the complexing of $\mathrm{Zn}$ ions with two cysteine residues (Cys 43 and 153) and one histidine residue (His 66). Cysteine residues are very susceptible to be oxidized by the photo-generated holes, leading to the loss of active site ( $\mathrm{Zn}$ ions) and the inactivation of YADH. After deposition of the titania nanoshell on the surface of the GCN core, YADH retained over $67 \%$ of its original activity (Figure 6g). The core@shell structure could then avoid the unfavorable contact between photo-generated holes of GCN and YADH, and then improve the compatibility of photocatalyst and YADH under light illumination. In short, the core@shell structure could not only coordinate the process of electron transfer and molecule diffusion, but also isolate the photo-generated holes and YADH, improving the compatibility between these two kinds of catalysts.

\section{Conclusions}

In summary, GCN@a-TiO 2 core@shell photocatalyst was prepared by coating an a- $\mathrm{TiO}_{2}$ nanoshell on the GCN core through a facile and green bioinspired mineralization method. The GCN core could be excited to generate electrons and holes under visible light illumination, whereas the porous, conductive $\mathrm{a}-\mathrm{TiO}_{2}$ nanoshell could rapidly extract the photo-generated electrons from GCN and allow the free diffusion of electron donor molecules to consume the photo-generated holes left on the GCN surface. The coordination between photo-generated electron transfer and electron donor molecule diffusion was realized by regulating the thickness of the a- $\mathrm{TiO}_{2}$ nanoshell. With the optimal nanoshell thickness, GCN@a- $\mathrm{TiO}_{2}-1$ exerted the 
highest photocatalytic NADH regeneration yield of $\sim 82.1 \%$, over two folds higher than GCN, which could be coupled with enzymatic reaction for stably and sustainable production of methanol. Hopefully, this study could provide a generic strategy for the design of high performance photocatalysts through the coordinated optimization of the transfer/diffusion of multiple substances/species.

\section{Associated content}

\section{Supporting Information}

FTIR spectra of GCN@a-TiO $2-1$, GCN and a-TiO ${ }_{2}$; High-resolution XPS spectra of C1s and N1s; The pore size distribution curves of GCN@a-TiO $-1, \mathrm{GCN} @ \mathrm{a}-\mathrm{TiO}_{2}-2$, GCN@a-TiO $2-3$, GCN and a-TiO 2 ; AFM images and corresponding height profiles of a- $\mathrm{TiO}_{2}-3, \mathrm{a}-\mathrm{TiO}_{2}-2, \mathrm{a}-\mathrm{TiO}_{2}-1$ and $\mathrm{a}-\mathrm{TiO}_{2}-1 *$; XPS valence band spectra of GCN,

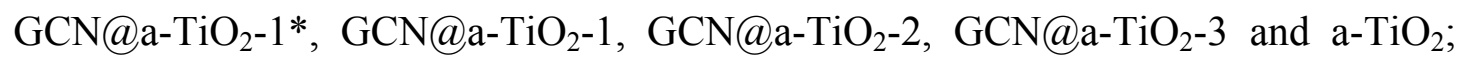
PL spectra at the excitation wavelength of $350 \mathrm{~nm}$ of $\mathrm{GCN} @ \mathrm{a}-\mathrm{TiO}_{2}-1$, GCN@PAH/Ti-1, GCN@Lys/Ti-1,GCN@Arg/Ti-1 and GCN; Energy Dispersive Spectroscopy (EDS) analysis of CdS@a- $\mathrm{TiO}_{2}-1$; The initial reaction rate (2 min) and balance yield of NADH regeneration with GCN@a-TiO $2-1$, GCN@PAH/Ti-1, GCN@Lys/Ti-1, GCN@Arg/Ti-1, GCN, CdS@a-TiO $2-1$ and CdS; EDS spectra of $\mathrm{ZnO} @ \mathrm{a}-\mathrm{TiO}_{2}$ and $\mathrm{SrTiO}_{3} @ \mathrm{a}-\mathrm{TiO}_{2}$; Photocatalytic NADH regeneration with $\mathrm{ZnO}$ and $\mathrm{ZnO} @ \mathrm{a}-\mathrm{TiO}_{2}$, and $\mathrm{SrTiO}_{3}$ and $\mathrm{SrTiO}_{3} @ \mathrm{a}-\mathrm{TiO}_{2} ;$ Steady-state PL spectra of GCN@a-TiO $2-1$, GCN@a-TiO $2-2$, GCN@a-TiO $2-3$ and GCN; UV-vis absorption of $0.05 \mathrm{w} / \mathrm{v} \%$ TEOA; UV-vis absorption of NADH regeneration solution before and after light illumination; Photocatalytic NADH regeneration and enzymatic 
degradation of the regenerated NADH; Photocatalytic NADH regeneration by GCN@a-TiO 2 under light-dark conditions; Transient absorption decay kinetics spectra of GCN@a-TiO 2 and [M] in PBS/TEOA (100 mM/15 w/v\%,pH=8.0) buffer with different ratio of $\mathrm{M}$ and $\mathrm{GCN} @ \mathrm{a}-\mathrm{TiO}_{2}$; Correlation between observed TAS decay kinetic constant (kobs) and ratio of $\mathrm{M}$ and $\mathrm{GCN} @ \mathrm{a}-\mathrm{TiO}_{2}$; Electron transfer efficiency and NADH regeneration yield with different ratio of $\mathrm{M}$ and $\mathrm{GCN} @ \mathrm{a}-\mathrm{TiO}_{2}$; Recycling stability of $\mathrm{GCN} @ \mathrm{a}-\mathrm{TiO}_{2}$ for $\mathrm{NADH}$ regeneration; XRD spectra of GCN@a-TiO ${ }_{2}$ before and after eight times recycling; TEM image of GCN@a-TiO before and after eight times recycling; Comparison of the conduction band (CB) and valence band (VB) potential of GCN with the redox potential of $\bullet \mathrm{OH} / \mathrm{H}_{2} \mathrm{O}$ and $\bullet \mathrm{O}_{2}{ }^{-} / \mathrm{O}_{2}$; ${ }^{1} \mathrm{H}$ NMR spectra of $\mathrm{NAD}^{+}$regeneration solution and freshly prepared $\mathrm{NAD}^{+}$solution; $\mathrm{NADH}$ regeneration enabled by $\mathrm{GCN} @ \mathrm{a}-\mathrm{TiO}_{2}-1$ under different protamine concentration; NADH regeneration enabled by $\mathrm{GCN} @ \mathrm{a}-\mathrm{TiO}_{2}-1$ under different pH values; $\mathrm{NADH}$ regeneration enabled by $\mathrm{GCN} @ \mathrm{a}-\mathrm{TiO}_{2}-1$ under different light intensity; UV-Vis diffuse reflectance spectra of $\mathrm{NAD}^{+}$and regenerated NADH; Chromatogram of methanol; The surface area and pore volume of GCN@a-TiO $2-1$, GCN@a-TiO $2-2, \mathrm{GCN} @ a-\mathrm{TiO}_{2}-3, \mathrm{GCN}$ and a-TiO ${ }_{2}$; The multi-exponential fitting results of time resolved photoluminescence.

\section{Author information}

\section{Corresponding Author}

Jiafu Shi (shijiafu@tju.edu.cn); Zhongyi Jiang (zhyjiang@tju.edu.cn).

\section{Present Addresses}


$\dagger$ Department of Engineering, Lancaster University, Lancaster LA1 4YW, United Kingdom; Email: xiaodong.wang@lancaster.ac.uk

\section{Notes}

All authors declare no competing financial interests.

\section{Acknowledgment}

This work was supported by the National Natural Science Foundation of China (21621004, 21776213, 91534126), Open funding supported by Guangdong Provincial Key Laboratory of New and Renewable Energy Research and Development (Y707s61001), National Key Laboratory of Biochemical Engineering (2015KF-03), and the Program of Introducing Talents of Discipline to Universities (B06006).

\section{References}

1. Xiao, M.; Wang, Z. L.; Lyu, M. Q.; Luo, B.; Wang, S. C.; Liu, G.; Cheng, H. M.; Wang, L. Z. Hollow Nanostructures for Photocatalysis: Advantages and Challenges. Adv. Mater. 2018, 31, 1801369.

2. Wang, S. B.; Lin, J. L.; Wang, X. C. Semiconductor-Redox Catalysis Promoted by Metal-Organic Frameworks for $\mathrm{CO}_{2}$ Reduction. Phys. Chem. Chem. Phys. 2014, 16, 14656-14660.

3. Lu, K. Q.; Xin, X.; Zhang, N.; Tang, Z. R.; Xu, Y. J. Photoredox Catalysis over Graphene Aerogel-Supported Composites. J. Mater. Chem. A. 2018, 6, 4590-4604. 
4. Wang, S. B.; Guan, B. Y.; Lu, Y.; Lou, X. W. Formation of Hierarchical $\mathrm{In}_{2} \mathrm{~S}_{3}$-CdIn $\mathrm{S}_{4}$ Heterostructured Nanotubes for Efficient and Stable Visible Light $\mathrm{CO}_{2}$ Reduction. J. Am. Chem. Soc. 2017, 139, 17305-17308.

5. Lin, X. H.; Gao, Y. L.; Jiang, M.; Zhang, Y. F.; Hou, Y. D.; Dai, W. X.; Wang, S. B.; Ding, Z. X. Photocatalytic $\mathrm{CO}_{2}$ Reduction Promoted by Uniform Perovskite Hydroxide $\mathrm{CoSn}(\mathrm{OH})_{6}$ Nanocubes. Appl. Catal. B Environ. 2018, 224, 1009-1016.

6. Wang, S. B.; Guan, B. Y.; Wang, X.; Lou, X. W. Formation of Hierarchical $\mathrm{Co}_{9} \mathrm{~S}_{8} @ \mathrm{ZnIn}_{2} \mathrm{~S}_{4}$ Heterostructured Cages as an Efficient Photocatalyst for Hydrogen Evolution. J. Am. Chem. Soc. 2018, 140, 15145-15148.

7. Qin, J. N.; Wang, S. B.; Wang, X. C. Visible-Light Reduction $\mathrm{CO}_{2}$ with Dodecahedral Zeolitic Imidazolate Framework ZIF-67 as an Efficient Co-catalyst. Appl. Catal. B Environ. 2017, 209, 476-482.

8. Chen, S. S.; Takata, T.; Domen, K. Particulate Photocatalysts for Overall Water Splitting. Nat. Rev. Mater. 2017, 2, 1-17.

9. Sun, M. H.; Huang, S. Z.; Chen, L. H.; Li, Y.; Yang, X. Y.; Yuan, Z. Y.; Su, B. L. Applications of Hierarchically Structured Porous Materials from Energy Storage and Conversion, Catalysis, Photocatalysis, Adsorption, Separation, and Sensing to Biomedicine. Chem. Soc. Rev. 2016, 45, 3479-3563. 
10. Luo, Z. S.; Fang, Y. X.; Zhou, M.; Wang, X. C. A Borocarbonitride Ceramic Aerogel for Photoredox Catalysis. Angew. Chem. Int. Ed. 2019, 58, 1-6.

11. Low, J. X.; Yu, J. G.; Jaroniec, M.; Wageh, S.; Al-Ghamdi, A. A. Heterojunction Photocatalysts. Adv. Mater. 2017, 29, 1601694.

12. Wang, H. L.; Zhang, L. S.; Chen, Z. G.; Hu, J. Q.; Li, S. J.; Wang, Z. H.; Liu, J. S.; Wang, X. C. Semiconductor Heterojunction Photocatalysts: Design, Construction, and Photocatalytic Performances. Chem. Soc. Rev. 2014, 43, $5234-5244$.

13. Li, W.; Elzatahry, A.; Aldhayan, D.; Zhao, D. Y. Core-Shell Structured Titanium Dioxide Nanomaterials for Solar Energy Utilization. Chem. Soc. Rev. 2018, 47, 8203-8237.

14. Takata, T.; Pan, C. S.; Nakabayashi, M.; Shibata, N.; Domen, K. Fabrication of a Core-Shell-Type Photocatalyst via Photodeposition of Group IV and V Transition Metal Oxyhydroxides: An Effective Surface Modification Method for Overall Water Splitting. J. Am. Chem. Soc. 2015, 137, 9627-9634.

15. Wu, Y. Z.; Ward-Bond, J.; Li, D. L.; Zhang, S. H.; Shi, J. F.; Jiang, Z. Y. g- $\mathrm{C}_{3} \mathrm{~N}_{4} @ \alpha-\mathrm{Fe}_{2} \mathrm{O}_{3} / \mathrm{C} \quad$ Photocatalysts: Synergistically Intensified Charge Generation and Charge Transfer for NADH Regeneration. ACS Catal. 2018, 8, $5664-5674$. 
16. Li, L. S.; Cao, R. G.; Wang, Z. J.; Li, J. J.; Qi, L. M. Template Synthesis of Hierarchical $\mathrm{Bi}_{2} \mathrm{E}_{3} \quad(\mathrm{E}=\mathrm{S}, \mathrm{Se}, \mathrm{Te})$ Core-Shell Microspheres and Their Electrochemical and Photoresponsive Properties. J. Phys. Chem. C. 2009, 113, 18075-18081.

17. Chen, S. S.; Shen, S.; Liu, G. J.; Qi, Y.; Zhang, F. X.; Li, C. Interface Engineering of a CoOx/ $/ a_{3} \mathrm{~N}_{5}$ Photocatalyst for Unprecedented Water Oxidation Performance under Visible-Light-Irradiation. Angew. Chem. Int. Ed. 2015, 54, 3047-3051.

18. Sun, D.; Jang, S.; Yim, S. J.; Ye, L.; Kim, D. P. Metal Doped Core-Shell Metal-Organic Frameworks@Covalent Organic Frameworks (MOFs@COFs) Hybrids as a Novel Photocatalytic Platform. Adv. Funct. Mater. 2018, 28, 1707110.

19. Han, S. C.; Pu, Y. C.; Zheng, L. X.; Zhang, J. Z.; Fang, X. S. Shell-Thickness Dependent Electron Transfer and Relaxation in Type-II Core-Shell CdS/TiO Structures with Optimized Photoelectrochemical Performance. J. Mater. Chem. A. 2015, 3, 22627-22635.

20. Zheng, Z. K.; Tachikawa, T.; Majima, T. Plasmon-Induced Spatial Electron Transfer Between Single Au Nanorods and ALD-coated $\mathrm{TiO}_{2}$ : Dependence on $\mathrm{TiO}_{2}$ Thickness. Chem. Commun. 2015, 51, 14373-14376. 
21. Lee, S.; Lee, K.; Kim, W. D.; Lee, S.; Shin, D. J.; Lee, D. C. Thin Amorphous $\mathrm{TiO}_{2}$ Shell on CdSe Nanocrystal Quantum Dots Enhances Photocatalysis of Hydrogen Evolution from Water. J. Phys. Chem. C. 2014, 118, 23627-23634.

22. Eisenberg, D.; Ahn, H. S.; Bard, A. J. Enhanced Photoelectrochemical Water Oxidation on Bismuth Vanadate by Electrodeposition of Amorphous Titanium Dioxide. J. Am. Chem. Soc. 2014, 136, 14011-14014.

23. Lee, C. Y.; Park, H. S.; Fontecilla-Camps, J. C.; Reisner, E. Photoelectrochemical $\mathrm{H}_{2}$ Evolution with a Hydrogenase Immobilized on a $\mathrm{TiO}_{2}$-Protected Silicon Electrode. Angew. Chem. Int. Ed. 2016, 55, 5971-5974.

24. Jiang, Y. J.; Yang, D.; Zhang, L.; Sun, Q. Y.; Sun, X. H.; Li, J.; Jiang, Z. Y. Preparation of Protamine-Titania Microcapsules Through Synergy Between Layer-by-Layer Assembly and Biomimetic Mineralization. Adv. Funct. Mater. 2009, 19, 150-156.

25. Liu, J.; Antonietti, M. Bio-inspired NADH Regeneration by Carbon Nitride Photocatalysis Using Diatom Templates. Energy Environ. Sci. 2013, 6, 1486-1493.

26. Zhang, S. H.; Shi, J. F.; Sun, Y. Y.; Wu, Y. Z.; Zhang, Y. S.; Cai, Z. Y.; Chen, Y. X.; You, C.; Han, P. P.; Jiang, Z. Y. Artificial Thylakoid for the Coordinated Photo-Enzymatic Reduction of Carbon Dioxide. ACS Catal. 2019, 9, 3913-3925. 
27. Bawazer, L. A.; Izumib, M.; Kolodinc, D.; Neilson, J. R.; Schwenzer, B.; Morse D. E. Evolutionary Selection of Enzymatically Synthesized Semiconductors from Biomimetic Mineralization Vesicles. Proc. Natl. Acad. Sci. USA 2012, 109, $1705-1714$.

28. Ran, J. R.; Guo, W. W.; Wang, H. L.; Zhu, B. C.; Yu, J. G.; Qiao, S. Z. Metal-Free 2D/2D Phosphorene/g- $\mathrm{C}_{3} \mathrm{~N}_{4}$ Van der Waals Heterojunction for Highly Enhanced Visible-Light Photocatalytic $\mathrm{H}_{2}$ Production. Adv. Mater. 2018, $30,1800128$.

29. Zhu, B. C.; Xia, P. F.; Ho, W. K.; Yu, J. G. Isoelectric Point and Adsorption Activity of Porous g-C $\mathrm{N}_{4}$. Appl. Surf. Sci. 2015, 344, 188-195.

30. Wang, X. C.; Maeda, K.; Thomas, A.; Takanabe, K.; Xin, G.; Carlsson, J. M.; Domen, K.; Antonietti, M. A Metal-Free Polymeric Photocatalyst for Hydrogen Production from Water under Visible Light. Nat. Mater. 2009, 8, 76-80.

31. Rahman, M. Z.; Tapping, P. C.; Kee, T. W.; Smernik, R.; Spooner, N.; Moffatt, J.; Tang, Y. H.; Davey, K.; Qiao, S. Z. A Benchmark Quantum Yield for Water Photoreduction on Amorphous Carbon Nitride. Adv. Funct. Mater. 2017, 27, 1702384.

32. Hu, S.; Shaner, M. R.; Beardslee, J. A.; Lichterman, M.; Brunschwig, B. S.; Lewis, N. S. Amorphous $\mathrm{TiO}_{2}$ Coatings Stabilize Si, GaAs, and Gap Photoanodes for Efficient Water Oxidation. Science 2014, 344, 1005-1009. 
33. Jun, Y. S.; Lee, E. Z.; Wang, X. C.; Hong, W. H.; Stucky, G. D.; Thomas, A. From Melamine-Cyanuric Acid Supramolecular Aggregates to Carbon Nitride Hollow Spheres. Adv. Funct. Mater. 2013, 23, 3661-3667.

34. Wang, S. B.; Guan, B. Y.; Lou, X. W. Construction of $\mathrm{ZnIn}_{2} \mathrm{~S}_{4}-\mathrm{In}_{2} \mathrm{O}_{3}$ Hierarchical Tubular Hetero-structures for Efficient $\mathrm{CO}_{2}$ Photoreduction. J. Am. Chem. Soc. 2018, 140, 5037-5040.

35. Wang, S. B.; Guan, B. Y.; Lou, X. W. Rationally Designed Hierarchical N-doped Carbon@ $\mathrm{NiCo}_{2} \mathrm{O}_{4}$ Double-Shelled Nanoboxes for Enhanced Visible Light $\mathrm{CO}_{2}$ Reduction. Energy Environ. Sci. 2018, 11, 306-310.

36. Ye, M. Y.; Zhao, Z. H.; Hu, Z. F.; Liu, L. Q.; Ji, H. M.; Shen, Z. R.; Ma, T. Y. 0D/2D Heterojunctions of Vanadate Quantum Dots/Graphitic Carbon Nitride Nanosheets for Enhanced Visible-Light-Driven Photocatalysis. Angew. Chem. Int. Ed. 2017, 56, 8407-8411.

37. Zhou, M.; Wang, S. B.; Yang, P. J.; Huang, C. J.; Wang, X. C. Boron Carbon Nitride Semiconductors Decorated with CdS Nanoparticles for Photocatalytic Reduction of $\mathrm{CO}_{2}$. ACS Catal. 2018, 8, 4928-4936.

38. Kang, S.; Huang, W.; Zhang, L.; He, M.; Xu, S.; Sun, D.; Jiang, X. Moderate Bacterial Etching Allows Scalable and Clean Delamination of $\mathrm{g}-\mathrm{C}_{3} \mathrm{~N}_{4}$ with Enriched Unpaired Electrons for Highly Improved Photocatalytic Water Disinfection. ACS Appl. Mater. Interfaces 2018, 10, 13796-13804. 
39. Tong, Z. W.; Yang, D.; Sun, Y. Y.; Nan, Y. H.; Jiang, Z. Y. Tubular $g-\mathrm{C}_{3} \mathrm{~N}_{4}$ Isotype Heterojunction: Enhanced Visible-Light Photocatalytic Activity through Cooperative Manipulation of Oriented Electron and Hole Transfer. Small 2016, $12,4093-4101$.

40. Lee, S. H.; Choi, D. S.; Kuk, S. K.; Park, C. B. Photobiocatalysis: Activating Redox Enzymes by Direct or Indirect Transfer of Photoinduced Electrons. Angew. Chem. Int. Ed. 2018, 57, 7958-7985.

41. Wang, X. D.; Saba, T.; Yiu, H. H. P.; Howe, R. F.; Anderson, J. A.; Shi, J. F. Cofactor NAD(P)H Regeneration Inspired by Heterogeneous Pathways. Chem 2017, 2, 621-654.

42. Yang, D.; Zhang, Y. S.; Zou, H. J.; Zhang, S. H.; Wu, Y. Z.; Cai, Z. Y.; Shi, J. F.; Jiang, Z. Y. Phosphorus Quantum Dots-Facilitated Enrichment of Electrons on g- $\mathrm{C}_{3} \mathrm{~N}_{4}$ Hollow Tubes for Visible-Light-Driven Nicotinamide Adenine Dinucleotide Regeneration. ACS Sustain. Chem. Eng. 2019, 7, 285-295.

43. Wang, X. L.; Li, Z.; Shi, J. F.; Wu, H.; Jiang, Z. Y.; Zhang, W. Y.; Song, X. K.; Ai, Q. H. Bioinspired Approach to Multienzyme Cascade System Construction for Efficient Carbon Dioxide Reduction. ACS Catal. 2014, 4, 962-972. 
TOC

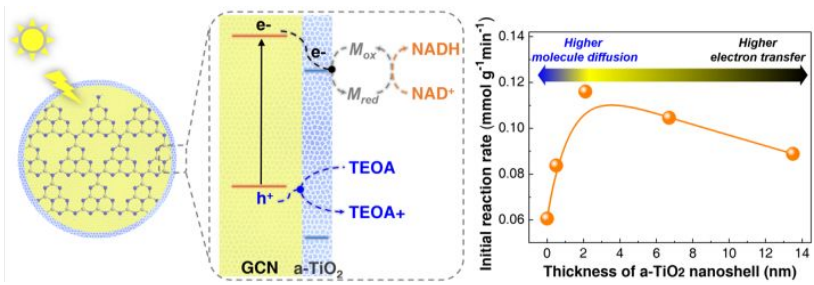

14

15

16

17 